\title{
ON FUZZY SUBBUNDLES OF VECTOR BUNDLES
}

\author{
V. MURALI and G. LUBCZONOK
}

Received 27 March 2002

\begin{abstract}
This paper considers fuzzy subbundles of a vector bundle. We define the operations sum, product, tensor product, Hom, and intersection of fuzzy subbundles and in each case, we characterize the corresponding flag of vector subbundles. We then propose two alternative definitions of integrability on fuzzy subbundles of a given type and discuss their naturality, merits, and shortcomings. We do these here with a view to introduce and study integrable fuzzy subbundles of tangent bundles on manifolds and foliations in further papers.
\end{abstract}

2000 Mathematics Subject Classification: 14F05, 14M15, 03E72, 14A20.

1. Introduction. A fuzzy subspace of a vector space can be characterized as a flag with weights, which are real numbers taken from the unit interval, attached at each component of the "nested" subspaces of the flag [7]. Similar ideas have been employed to study fuzzy subgroups of finite Abelian groups by Murali and Makamba [14]. A number of authors [1, 2, 5, 6, 8, 12] have considered fuzzy subspaces of vector spaces in other contexts. On the other hand, flags and flag manifolds are familiar objects of study for algebraists, geometers, topologists, and others $[3,4,11,13]$. The present work was suggested by analogy with basic constructions of vector bundles. A fuzzy subbundle is a complex mathematical construction. It consists of a chain of subbundles and weights. Chains of subbundles or foliations are considered and investigated in foliation theory, for example, see [10]. In this context, we will have to deal with weights which are new components in the case of fuzzy subbundles. It is worthwhile to investigate the role and possible applications of weights. Therefore, a large part of this paper is devoted to the formulation and elucidation of definitions and their simple consequences. Further, we propose some integrability conditions on fuzzy subbundles, basically from an algebraic point of view. In so doing, we hope to lay the foundations for the study of foliations and other geometric aspects of fuzzy subbundles. We will omit the standard topological considerations in this paper, but concentrate largely on algebraic aspects.

\section{Preliminaries}

2.1. Definitions. In this paper, $V$ denotes a vector space over the field of real numbers of dimension $n<\infty$. Further, suppose that the triple $\xi=(E, M, p)$ is a vector bundle, with $p: E \rightarrow M$ being the projection of the total space $E$ onto 
the base space $M$. For every $x \in M$, the fiber $E_{x}=p^{-1}(x)$ is a vector space over $F$ isomorphic to $V$. For the purposes of formal constructions, it is not necessary to impose local triviality condition on the vector bundle, and as such, we have not assumed any such condition in general, unless otherwise stated in a particular context. We use $I=[0,1]$, the real unit interval as a chain with the usual ordering in which $\wedge$ stands for infimum (or intersection) and $\vee$ stands for supremum (or union).

Definition 2.1. A fuzzy subbundle of $\xi$ is a fuzzy subset $\mu: E \rightarrow I$ such that the following holds. For $x \in M$, the restriction $\mu_{x}: E_{x} \rightarrow I$ of $\mu$ to $E_{x}$ is a fuzzy subspace of $E_{x}$, which means that for every $x \in M$ and $u, v \in E_{x}$, $\mu_{x}(\alpha u+\beta v) \geq \mu(u)_{x} \wedge \mu_{x}(v)$, for $\alpha, \beta \in F$.

Without loss of generality, throughout this paper we tacitly assume that $\mu_{x}(0)=1$ for every fuzzy subspace $\mu_{x}, x \in M$. We will use the following form of Lowen's representation theorem [6].

THEOREM 2.2. A fuzzy subset $\mu$ of $V$ is a fuzzy subspace of $V$ if and only if there exist an integer $k \leq n$ and weights $1=\alpha_{0}>\alpha_{1}>\cdots>\alpha_{k} \geq 0$, and $a$ strict flag $V^{\alpha_{0}} \subset V^{\alpha_{1}} \subset \cdots \subset V^{\alpha_{k}}=V$ such that $\mu \mid V^{\alpha_{j}} \backslash V^{\alpha_{j-1}}=\alpha_{j}$ for $1 \leq j \leq k$.

Therefore, for every $x \in M$, we have a strict flag

$$
V_{x}^{\alpha_{0}(x)} \subset V_{x}^{\alpha_{1}(x)} \subset \cdots \subset V_{x}^{\alpha_{k}(x)} \subset E_{x},
$$

where $k$ may depend on $x$ and

$$
1=\alpha_{0}(x)>\alpha_{1}(x)>\cdots>\alpha_{k}(x) \geq 0, \quad k \leq n,
$$

are weights. Denote

$$
C_{x}{ }^{\alpha_{j}(x)}=V_{x}{ }^{\alpha_{j}(x)} \backslash V_{x}{ }^{\alpha_{j-1}(x)}, \quad j=1, \ldots, k, x \in M .
$$

Clearly,

$$
C_{x}{ }^{\alpha_{j}(x)}=\mu_{x}^{-1}\left(\alpha_{j}(x)\right), \quad j=1, \ldots, k, x \in M
$$

REMARK 2.3. In this paper, we assume that the number of subspaces $k$ in the flag at $x \in M$ is independent of $x$. Thus each weight $\alpha_{j}(x), j=0,1, \ldots, k$, defines a fuzzy subset of $M$.

From the definition of fuzzy subbundle and the Lowen's representation theorem as stated above, we have the following consequences, which we state as a proposition.

Proposition 2.4. Suppose $\xi=(E, p, M)$ is a vector bundle. A fuzzy subset $\mu: E \rightarrow I$ is a fuzzy subbundle if and only if there exist

(i) a flag on $E_{x}$ for every $x \in M$, associated with $\mu_{x}$, 
(ii) a finite chain of fuzzy subsets defined on $M$ in the form of

$$
1=\alpha_{0}(x)>\alpha_{1}(x)>\cdots>\alpha_{k}(x) \geq 0, \quad k \leq n, x \in M .
$$

In fuzzy set theory, it is not uncommon to discuss definitions in terms of the so-called levels. That is, the $c$-cut levels for $0 \leq c \leq 1$ of a fuzzy subbundle is a crisp subbundle. The following example demonstrates that if $c$-cuts are crisp subbundles, then the weights $\alpha$ 's are constants.

EXAMPLE 2.5. Let $\xi^{\prime}=\left(E^{\prime}, p^{\prime}, M\right)$ be a subbundle of $\xi=(E, p, M)$. Thus $E_{x}^{\prime} \subset$ $E_{x}$ is a subspace, $\operatorname{dim} E_{x}^{\prime}=m, m<n$. Consider a fuzzy subbundle, with the flag

$$
\{0\} \subset E_{x}^{\prime} \subset E_{x}
$$

and the weights

$$
1>\alpha(x)>0, \quad x \in M
$$

Thus, given $c \in I$, the $c$-cut consists of $E_{x}^{\prime}$ if $\alpha(x) \geq c$ and $\{0\}$ if $\alpha(x)<c$. We see that every $c$-cut of $\mu$ is a subbundle of $\xi$ only if $\alpha(x)$ is a constant function on $M$.

For this reason, we proposed the more general definition as given in Definition 2.1, from which the following is, however, immediate.

For every $j$, the set of all $v \in E$ such that $\mu(v) \geq \alpha_{j}(p(v))$ is a subbundle of $\xi$ if $\operatorname{dim} V_{x}{ }^{\alpha_{j}(x)}$ is constant for $x \in M$.

2.2. Morphisms. For the sake of completeness, we recall the following.

(i) If $\phi: V \rightarrow W$ is a linear map and if $\mu$ is a fuzzy subspace of $V$, then by the image of the fuzzy subspace $\mu$, we mean the fuzzy subset $\phi(\mu)$ of $W$ defined by $(\phi(\mu))(y)=\sup \{\mu(x): \phi(x)=y\}$ if $\phi^{-1}(y) \neq \varnothing$, and $(\phi(\mu))(y)=0$ if $\phi^{-1}(y)=\varnothing$. It is easy to check that $\phi(\mu)$ is a fuzzy subspace of $W$ whenever $\mu$ is a fuzzy subspace of $V$.

(ii) A pair $(\phi, f)$, where

$$
\phi: E \longrightarrow E^{\prime}, \quad f: M \longrightarrow M^{\prime},
$$

is a morphism between two vector bundles $\xi=(E, p, M)$ and $\xi^{\prime}=\left(E^{\prime}, p^{\prime}, M^{\prime}\right)$ if $f \circ p=p^{\prime} \circ \phi$ and the restriction $\phi_{x}$ of $\phi$ to $E_{x} \rightarrow E_{f(x)}^{\prime}$ is linear for every $x \in M$. For further results, we refer the reader to [4].

Given a fuzzy subbundle $\nu$ of $\xi^{\prime}$, we can induce a fuzzy subbundle $\mu$ of $\xi$ by the composition

$$
\mu=\phi \circ \nu
$$


Clearly it is a pullback operation, and for every $x \in M$,

$$
\mu_{x}=\phi_{x} \circ v_{f(x)}
$$

We can also define a push-forward operation (which is not possible for the case of crisp subbundles) on fuzzy subbundles as follows.

Let $\mu$ be a fuzzy subbundle of $\xi$. Denote by $\phi_{x}\left(\mu_{x}\right)$ the image of the fuzzy subspace $\mu_{x}$. It is a fuzzy subspace of $E_{f(x)}^{\prime}$ [8]. Given $y \in M^{\prime}$, we define

$$
v_{y}=\inf \left\{\phi_{x}\left(\mu_{x}\right): x \in f^{-1}(y)\right\}
$$

Then the collection $v=\left\{v_{y}: y \in M^{\prime}\right\}$ of fuzzy subspaces defines a fuzzy subbundle of $\xi^{\prime}$. We call this subbundle $v$ the image of $\mu$ by the morphism $(\phi, f)$.

2.3. Fuzzy subbundles as sections. We will give a description of fuzzy subbundle as a section of crisp bundle. Recall that $E_{x}$ is vector space isomorphic to a given vector space $V$ over a field $F$, for $x \in M$. Denote by $F(V)$ the set of all fuzzy subspaces of $V$. Then we construct the bundle $F(\xi)$ over $M$ with the total space $F(E)=\bigcup\left\{F\left(E_{\chi}\right): x \in M\right\}$ and the projection $F(p): F(E) \rightarrow M$, where $F(p)(\mu)=x$ if $\mu \in F\left(E_{x}\right)$. Any section $\mu$ of $F(\xi)$ defines a fuzzy subbundle of $\xi$ with $\mu_{x}=\mu(x) \in F\left(E_{x}\right), x \in M$. This construction becomes more explicit for a weighted flag of a given type of a vector space of finite dimension over the field $F$, where $F$ is the field of real or complex numbers. We will now explain the concept of "type" as follows.

Let $l=\left(l_{1}, l_{2}, \ldots, l_{k}\right)$ be a finite sequence of positive integers such that $0<$ $l_{1}<l_{2}<\cdots<l_{k}$. A fuzzy subspace $\mu$ is said to be of type $l$ if its flag contains $k$ subspaces $V_{1}, V_{2}, \ldots, V_{k}$ with $\operatorname{dim} V_{p}=l_{p}, p=1,2, \ldots, k$. Denote by $F_{l} V(l)$ the set of all fuzzy subspaces of $V$ of type $l$. We often identify type $l$ of a flag simply with $l=\left(l_{1}, l_{2}, \ldots, l_{k}\right)$ where the various $l_{i}$ 's are the dimensions of the various subspaces in $V$. If we identify $V$ with $F^{n}$, then clearly the orthogonal group $\mathrm{SO}(n, \mathbb{R}),(\mathrm{SU}(n)$ in the case of complex vector space) acts transitively on the flags of the type $l$. Hence the set of all flags of type $l$ is a homogeneous manifold denoted by $F L\left(l, F^{n}\right)$.

To discuss the weights attached to these subspaces of a flag associated with a fuzzy subspace $\mu$, consider the open simplex in $(0,1)^{k}$, denoted by $\triangle_{k}$ of all finite sequences $\alpha_{1}, \alpha_{2}, \ldots, \alpha_{k}$ of real numbers of the form $1>\alpha_{1}>\alpha_{2}>\cdots>$ $\alpha_{k}>0$. With these notations, we can identify

$$
F V(l)=F L\left(l, F^{n}\right) \times \triangle_{k}
$$

as follows (see (2.1)). Every $\mu$ can be identified with

$$
\{0\} \subset V_{1}^{\alpha_{1}} \subset \cdots \subset V_{k}{ }^{\alpha_{k}} \subset V,
$$


which gives rise to a flag

$$
\{0\} \subset V_{1} \subset \cdots \subset V_{k} \subset V,
$$

and the sequence $\alpha_{1}, \alpha_{2}, \ldots, \alpha_{k}$ in

$$
1>\alpha_{1}>\alpha_{2}>\cdots>\alpha_{k}>0,
$$

and vice versa.

Let, as before, $\xi=(E, M, p)$ be a vector bundle.

Suppose

$$
F_{l}(E)=\bigcup_{x \in M} F E_{x}(l),
$$

where $F E_{x}(l)$ is the set of all fuzzy subspaces of $E_{x}$ of type $l$. Now, we can construct a new fiber bundle $F \xi=\left(F_{l}(E), M, F(p)\right)$ of fuzzy subspaces of a given type $l$ and $F(p): F_{l}(E) \rightarrow M$ is the fiber bundle projection. Then, we can regard any fuzzy subbundle of $\xi$ as a section of the fiber bundle $F \xi$, that is, a mapping $\mu: M \rightarrow F_{l}(E)$ such that $\mu \circ F(p)=$ identity on $M$.

Suppose now $\xi=(E, M, p)$ is locally trivial. Let $\phi_{U}:\left.E\right|_{U} \rightarrow U \times F^{n}$ be a local trivialization of $\xi$, where $U \subset M$ is open, $\left.E\right|_{U}=p^{-1}(U)$. Then $\mu_{U}=\mu \circ \phi_{U}^{-1}$ is a fuzzy subbundle of the trivial bundle $U \times F^{n}$. If $\phi_{V}:\left.E\right|_{V} \rightarrow V \times F^{n}$ is another local trivialization of $\xi$, then $\phi_{V} \circ \phi_{U}{ }^{-1}$ restricted to $U \cap V$ defines an isomorphism of the trivial bundle $(U \cap V) \times F^{n}$, and therefore

$$
\phi_{V} \circ \phi_{U}^{-1}(x, v)=\left(x, \phi_{V U}(x) v\right), \quad x \in U \cap V, v \in F^{n},
$$

where $\phi_{V U}: U \cap V \rightarrow \mathrm{GL}\left(F^{n}\right)$ is the transition function [4]. We have $\mu_{U}=\mu_{V}$ 。 $\phi_{V U}$ on $U \cap V$. We note that if $E$ is locally trivial, then so is $F \xi$. Given a local trivialization $\left.E\right|_{U} \rightarrow U \times F^{n}$, we have the induced trivialization $\left.F_{l}(E)\right|_{U} \rightarrow U \times F V(l)$, where $V=F^{n}$. The $\mu$-section induces on every trivialization a corresponding section $\mu_{U}: U \rightarrow F V(l)$, where $V=F^{n}$. This point of view will be utilized later.

2.4. Continuity of fuzzy subbundles. In this section, we make a few observations about continuity of fuzzy subbundles. Given $E_{x}, x \in M, \mu_{x}: E_{x} \rightarrow I$ is a step function with finite number of values in $I$ and is only right continuous. We want to define a continuous dependence of $\mu_{x}$ on $x \in M$. Suppose $E, M$ are $C^{\infty}$ manifolds and $p: E \rightarrow M$ is a $C^{\infty}$ map. We consider the example of trivial bundle case in the next proposition.

Proposition 2.6. Suppose $p: U \times \mathbb{R}^{n} \rightarrow U$, where $U$ is an open connected subset in $\mathbb{R}^{m}$ and $p$ is the trivial bundle projection and a fuzzy subbundle $\mu$ : $U \times \mathbb{R}^{n} \rightarrow I$ is of a given type l. Given a fixed $v \in \mathbb{R}^{n}$, if $x \rightarrow \mu(x, v)$ is continuous on $U$, then the flag for $\mu$ is constant and the weights are continuous functions on $U$. 
Here we understand the flag for $\mu$ being constant in the sense that for every $x \in U, V_{j}{ }^{\alpha_{j}(x)}=V_{j}, j=1,2, \ldots, k$, where $V_{j}$ is a fixed subspace of $\mathbb{R}^{n}$.

Proof. Let $\alpha_{1}\left(x_{0}\right)<\alpha_{2}\left(x_{0}\right)<\cdots<\alpha_{k}\left(x_{0}\right)$ be the weights at $x_{0} \in U$. Consider a family of disjoint neighborhoods $I_{1}, I_{2}, \ldots, I_{k}$ of $\alpha_{1}\left(x_{0}\right), \alpha_{2}\left(x_{0}\right), \ldots$, $\alpha_{k}\left(x_{0}\right)$, respectively. Given $j$, choose any $v_{j} \in \mathbb{R}^{n}$ such that

$$
\mu\left(x_{0}, v_{j}\right)=\alpha_{j}\left(x_{0}\right), \quad \text { for } j=1,2, \ldots, k
$$

By assumption,

$$
\mu\left(x, v_{j}\right) \longrightarrow \mu\left(x_{0}, v_{j}\right)=\alpha_{j}^{\prime}\left(x_{0}\right), \text { for } j=1,2, \ldots, k \text {, as } x \longrightarrow x_{0}
$$

Hence there is a neighborhood $N$ of $x_{0}$ such that $\mu\left(x, v_{j}\right) \in I_{j}$ for $j=1,2, \ldots, k$. Since $\mu(x, \cdot)$ has exactly $k$ values and $I_{j}$ 's are disjoint, we must have

$$
\mu\left(x, v_{j}\right)=\alpha_{j}(x), \quad \text { for } j=1,2, \ldots, k
$$

This shows that $\alpha_{j}(x) \rightarrow \alpha_{j}\left(x_{0}\right)$ as $x \rightarrow x_{0}$. In other words, $\alpha_{j}(x)$ is continuous for $j=1,2, \ldots, k$. Now we will demonstrate that

$$
V_{x}^{\alpha_{j}(x)}=V_{j} \subset \mathbb{R}^{n}, \quad \text { for } j=1,2, \ldots, k
$$

Consider a fixed point $x_{0} \in U$ and choose a $v \in \mathbb{R}^{n}$. Then $\mu\left(x_{0}, v\right)=\alpha_{i}\left(x_{0}\right)$ for some $1 \leq i \leq k$. From the proof of continuity of the weights $\alpha$ 's, we conclude that there is a neighborhood $N$ of $x_{0}$ in which $\mu(x, v)=\alpha_{i}(x)$. Now let $v \in$ $V_{x_{0}}^{\alpha_{j}\left(x_{0}\right)}$, that is, $\mu\left(x_{0}, v\right)=\alpha_{i}\left(x_{0}\right)$ for some $i \geq j$. Therefore, $\mu(x, v)=\alpha_{i}(x) \geq$ $\alpha_{j}(x)$. In other words, $v \in V_{x}^{\alpha_{j}(x)}$ in some neighborhood of $x_{0}$. Since the role $x$ and $x_{0}$ can be reversed, we have

$$
V_{x}^{\alpha_{j}(x)}=V_{x_{0}}^{\alpha_{j}\left(x_{0}\right)}, \text { for } x \in U
$$

in some neighborhood of $x_{0}$ and $j=1,2, \ldots, k$. It shows that the set of $x$ satisfying (2.22) is open. It is trivial that this set is also closed from the assumption of continuity of $\mu$ and proven continuity of weights. Since $U$ is connected,

$$
V_{x}^{\alpha_{j}(x)}=V_{x_{0}}^{\alpha_{j}\left(x_{0}\right)}=V_{j}, \quad \forall x \in U, j=1,2, \ldots, k
$$

This completes the proof.

DEFINITION 2.7. A fuzzy subbundle of a given type $l=\left(l_{1}, l_{2}, \ldots, l_{k}\right)$ is continuous or differentiable $\left(C^{\infty}\right)$ if the corresponding section $s_{\mu}: M \rightarrow F \xi$ is continuous or differentiable $\left(C^{\infty}\right)$. 
Clearly this definition means the following.

(i) Every distribution of the flag for $\mu$ is continuous, or differentiable $\left(C^{\infty}\right)$.

(ii) Every weight is a continuous, or differentiable function $\left(C^{\infty}\right)$ on $M$.

3. Operations on fuzzy subbundles. All the operations on fuzzy subbundles are done pointwise, that is, we perform the corresponding operations on fuzzy subspaces of $E_{x}$ at every $x \in M$. Thus the properties of the operations on fuzzy subbundles follow from the corresponding properties of operations on fuzzy subspaces [7]. We would like to point out that the resulting fuzzy subbundles in various cases may produce flags with the number of subspaces varying with $x$ even in the cases of fuzzy subbundles of constant type. This anomaly exists even in the classical cases as most of the operations do not give rise to further subbundles although they do give subsheaves. The actual calculations of the number of weights and the weights themselves in various cases are given in [7].

3.1. Intersection, sum, and dual of fuzzy subbundles. Let $\mu$ and $\nu$ be fuzzy subbundles of a vector bundle $\xi=(E, p, M)$. Let

$$
\begin{aligned}
& \left\{0_{x}\right\} \subset V_{x}{ }^{\alpha_{1}(x)} \subset \cdots \subset V_{x}{ }^{\alpha_{k}(x)} \subset E_{x}, \\
& \left\{0_{x}\right\} \subset W_{x}{ }^{\beta_{1}(x)} \subset \cdots \subset W_{x}{ }^{\beta_{l}(x)} \subset E_{x}
\end{aligned}
$$

be the flags and weights of $\mu_{x}$ and $v_{x}$, respectively, for a given $x \in M$.

Fuzzy intersection is given by the equation $(\mu \wedge \nu)_{x}=\mu_{x} \wedge \nu_{x}, x \in M$.

A number $\gamma^{*}(x)=\min \left\{\alpha_{r}(x), \beta_{s}(x)\right\}$ is a weight for $(\mu \wedge \nu)_{x}$ if

$$
\begin{gathered}
\alpha_{r-1}(x)>\gamma^{*}(x) \geq \alpha_{r}(x), \quad \beta_{s-1}(x)>\gamma^{*}(x) \geq \beta_{s}(x), \\
U^{\gamma^{*}(x)}=V^{\alpha_{r}(x)} \cap W^{\beta_{s}(x)} \neq\left\{0_{x}\right\}, \quad x \in M .
\end{gathered}
$$

Fuzzy sum is defined by

$$
(\mu+v)_{x}(v)=\left(\mu_{x}+v_{x}\right)(v)=\bigvee\left\{\mu_{x}(v-t) \wedge v_{x}(t): t \in E_{x}\right\}
$$

A number $\gamma^{*}(x)=\min \left\{\alpha_{r}(x), \beta_{s}(x)\right\}$ is a weight for $(\mu+\nu)_{x}$ if

$$
\alpha_{r-1}(x)>\gamma^{*}(x) \geq \alpha_{r}(x), \quad \beta_{s-1}(x)>\gamma^{*}(x) \geq \beta_{s}(x) .
$$

The corresponding subspace of $E_{x}$ for $x \in M$ is

$$
U^{\gamma^{*}(x)}=V^{\alpha_{r}(x)}+W^{\beta_{s}(x)} .
$$

Dual subbundle $\mu^{*}$ is a fuzzy subbundle of the dual bundle $\xi^{*}$, the fiber at $x \in M$ is $\left(\mu^{*}\right)_{x}=\left(\mu_{x}\right)^{*}$, where for $f \in\left(E_{x}\right)^{*}$,

$$
\left(\left(\mu_{x}\right)^{*}\right)(f)= \begin{cases}1, & f=0, \\ 1-\sup \left\{\mu_{x}(t): t \in E_{x}, f(t) \neq 0\right\}, & f \neq 0 .\end{cases}
$$


Then the weights for $\mu^{*}$ are $\alpha_{j}{ }^{*}(x)=1-\alpha_{j}(x)$, with the corresponding subspaces

$$
V^{\alpha_{j}{ }^{*}(x)}=\left(V^{\alpha_{j}(x)}\right)^{0}=\text { the annihilator of } V^{\alpha_{j}(x)}, \quad x \in M
$$

3.2. Whitney sum and tensor product. Let $\xi=(E, p, M)$ and $\xi^{\prime}=\left(E^{\prime}, p^{\prime}, M\right)$ be two vector bundles over $M$. Let $\mu$ and $\nu$ be two fuzzy subbundles of $\xi$ and $\xi^{\prime}$, respectively.

Consider the Whitney sum $\xi \oplus \xi^{\prime}$, where the total space of $\xi \oplus \xi^{\prime}$ is the union of $E_{x} \oplus E_{x}^{\prime}, x \in M$. The Whitney sum $\mu \oplus v$ is a fuzzy subbundle of $\xi \oplus \xi^{\prime}$, with $(\mu \oplus \nu)_{x}=\mu_{x} \oplus \nu_{x}, x \in M$, where $\mu_{x} \oplus \nu_{x}$ is the fuzzy subspace of $E_{x} \oplus E_{x}^{\prime}$ defined by $\left(\mu_{x} \oplus v_{x}\right)\left(t, t^{\prime}\right)=\mu_{x}(t) \wedge v_{x}\left(t^{\prime}\right)$ for $t \in E_{x}, t^{\prime} \in E_{x}^{\prime}$. Accordingly, the number $\gamma^{*}(x)=\min \left\{\alpha_{r}(x), \beta_{s}(x)\right\}$ is a weight for $(\mu \oplus \nu)_{x}$ if and only if the rectangle

$$
\left[\alpha_{r}(x), \alpha_{r-1}(x)\right] \times\left[\beta_{s}(x), \beta_{s-1}(x)\right]
$$

has nonempty intersection with the diagonal $I \times I$. The corresponding subspace is

$$
V^{\gamma(x)} \oplus W^{\gamma(x)} \subset E_{x} \oplus E_{x}^{\prime}
$$

Similarly, for the tensor product, $(\mu \otimes v)_{x}=\mu_{x} \otimes v_{x}$ is a fuzzy subspace of $E_{x} \otimes E_{x}^{\prime}$, for $x \in M$ see [7, Section 4.2, page 204]. Accordingly, the number $\gamma^{*}(x)=\min \left\{\alpha_{r}(x), \beta_{s}(x)\right\}$ is a weight for $(\mu \oplus \nu)_{x}$ if and only if the rectangle

$$
\left[\alpha_{r}(x), \alpha_{r-1}(x)\right] \times\left[\beta_{s}(x), \beta_{s-1}(x)\right]
$$

has nonempty intersection with the diagonal $I \times I$.

3.3. Bundle of bilinear forms and Hom bundles. Let $\xi=(E, p, M)$ and $\xi^{\prime}=\left(E^{\prime}, p^{\prime}, M\right)$ be two vector bundles over $M$. Let $\mu$ and $v$ be two fuzzy subbundles of $\xi$ and $\xi^{\prime}$, respectively. Consider the bundle $B\left(\xi, \xi^{\prime}\right)=\left(B\left(E, E^{\prime}\right), \tilde{p}, M\right)$, where $B\left(E, E^{\prime}\right)=\bigcup\left(B\left(E_{x}, E_{x}^{\prime}\right): x \in M\right)$ with the fiber being the space of all bilinear maps from $E_{x} \times E_{x}^{\prime} \rightarrow F$. Given two fuzzy subbundles $\mu, v$ of $\xi$ and $\xi^{\prime}$, respectively, we define a fuzzy subbundle of $B\left(\xi, \xi^{\prime}\right)$ as follows:

$$
(\mu \cdot v)_{x}=\mu_{x} \cdot v_{x}, \quad x \in M
$$

as given in [7, Section 5.1, page 205]. The corresponding flag for $\mu_{x} \cdot v_{x}$ is given by

$$
B^{\alpha}\left(E_{x}, E_{x}^{\prime}\right)=\left\{\phi \in B\left(E_{x}, E_{x}^{\prime}\right): \phi(u, v)=0 \text { for }(u, v) \in E_{x}^{1-\alpha} \times E_{x}^{\prime 1-\alpha}\right\} .
$$

Similarly, for the bundle $\operatorname{Hom}\left(\xi, \xi^{\prime}\right)$ with the fibers,

$$
\operatorname{Hom}_{x}\left(\xi, \xi^{\prime}\right)=\operatorname{Hom}\left(E_{x}, E_{x}^{\prime}\right), \quad x \in M .
$$


We have $\operatorname{Hom}(\mu, v)$ a fuzzy subbundle of $\operatorname{Hom}\left(\xi, \xi^{\prime}\right)$ with the subspaces

$$
\left(E_{x}^{1-\alpha}\right)^{\perp} \otimes\left(E_{x}^{\prime}\right)^{\alpha}, \quad x \in M .
$$

Accordingly, a number $\gamma^{*}(x)=\min \left\{\alpha_{r}(x), \beta_{s}(x)\right\}$ is a weight for $\operatorname{Hom}\left(\mu_{x}, \nu_{x}\right)$ if and only if the rectangle

$$
\left[\alpha_{r}(x), \alpha_{r-1}(x)\right] \times\left[\beta_{s}(x), \beta_{s-1}(x)\right]
$$

has nonempty intersection with the diagonal $I \times I$. Then

$$
\operatorname{Hom}^{\gamma^{*}}\left(\mu_{x}, v_{x}\right)=\left(E_{x}{ }^{1-\alpha_{r}(x)}\right)^{\perp} \otimes\left(E_{x}^{\prime}\right)^{\beta_{s}(x)}, \quad x \in M .
$$

4. Subbundles of tangent bundles. In this section, we consider fuzzy subbundles of tangent bundle on a manifold and we propose two different definitions of integrability of fuzzy subbundles. These two definitions apply to fuzzy subbundles $\mu$ of $T M$ of a given type $l$, where $T M$ is the tangent bundle of a manifold $M$.

4.1. Integrability I. We firstly recall that by a distribution of a tangent bundle $T M$ of a manifold $M$, we mean a field of subspaces $\left\{D_{x}\right\}$, where $D_{x} \subset T_{x} M$ for $x \in M$ and $\operatorname{Dim}\left(D_{x}\right)=k$, where $k$ is independent of $x \in M$ [10]. It can be regarded as a section of the Grassmanian bundle $G_{k}(M)$, [10]. In terms of section of Grassmanian bundle, integrability means existence of local coordinates in which the section is constant. In the context of fuzzy subbundle of type $l$, it works as follows.

Let $\mu$ be a fuzzy subbundle of the tangent bundle $T=(T M, M, p)$ where $p: T M \rightarrow M$ is the bundle projection. It defines a section $\mu$ of the bundle $F(T)=$ $\{F L(T M), M, F(p)\}$. Every local coordinate about $x \in M$ defines a trivialization of $\left.T M\right|_{U} \rightarrow U \times \mathbb{R}^{n}$ and the corresponding trivialization of $\left.F L(T M)\right|_{U} \rightarrow U \times$ $F \mathbb{R}^{n}(l)$. This allows us to consider the induced section $\left.\mu\right|_{U}: U \rightarrow F \mathbb{R}^{n}(l)$ as discussed in the end of Section 2. We say that $\mu$ is integrable if about every $x \in M$ there is a neighborhood $U$ of $x$ and coordinates in $U$ such that the induced section $\left.\mu\right|_{U}$ is constant.

This definition, among other things, implies that

(i) the flag for $\mu$ is integrable or all its distributions of subspaces are integrable,

(ii) weights are constant on $U$.

We observe that the above definition, though a natural one, is too restrictive because we would like to have a definition of integrability that would be equally applicable to fuzzy subbundles with variable weights. This is the content of next two subsections. These concepts of integrability depend on what we mean by isomorphism of two fuzzy subspaces.

4.2. Integrability II. Let $(U, \mu)$ and $(V, \nu)$ be two fuzzy subspaces. We recall that a linear isomorphism of vector spaces $L: U \rightarrow V$ is an isomorphism of 
fuzzy subspaces if

$$
v(L(u))=\mu(u), \quad u \in U
$$

Suppose $\mu$ is a fuzzy subbundle of the tangent bundle TM. A diffeomorphism $f: M \rightarrow M$ induces a diffeomorphism $d f: T M \rightarrow T M$ through the differential $d f$ of $f$. We say that $f$ preserves $\mu$ if

$$
\mu(\xi)=\mu(d f(\xi)), \quad \text { for } \xi \in T M .
$$

Equivalently, if $x \in M$ and $\xi \in T_{x} M$, then

$$
\mu_{x}(\xi)=\mu_{f(x)}(d f(x)(\xi))
$$

which means that the differential

$$
d f(x): T_{x} M \longrightarrow T_{f(x)} M
$$

is an isomorphism of fuzzy subspaces $\mu_{x}$ and $\mu_{f(x)}$. Further, we notice that

$$
\begin{gathered}
\alpha_{j}(x)=\alpha_{j}(f(x)), \quad \forall x \in M, j=1,2, \ldots, k, \\
d f(x)\left(V_{x}^{\alpha_{j}(x)}\right)=V_{f(x)}{ }^{\alpha_{j}(f(x))}=V_{f(x)}{ }^{\alpha_{j}(x)}
\end{gathered}
$$

since (4.5) is true.

Conversely, we have the following immediately straightforward result.

Proposition 4.1. A diffeomorphism $f: M \rightarrow M$ preserves a fuzzy subbundle $\mu: T M \rightarrow I$ if and only if $f$ satisfies (4.5) and (4.6).

Similarly, if $X$ is a vector field on $M$ and $\left\{\phi_{t}\right\}$, the corresponding flow for $X$, then we have the flow

$$
\tilde{\phi}_{t}=d \phi_{t}: T M \longrightarrow T M
$$

and we say $X$ preserves $\mu$ if $\left\{\phi_{t}\right\}$ preserves $\mu$ for all $t \in \mathbb{R}$.

From (4.5) and (4.6),

$$
\alpha_{j}(x)=\alpha_{j}\left(\phi_{t}(x)\right)
$$

(or each weight $\alpha_{j}$ is a first integral of $X$ ),

$$
\tilde{\phi}_{t}\left(V_{x}{ }^{\alpha_{j}(x)}\right)=V_{\phi_{t}(x)}^{\alpha_{j}(x)}, \quad \forall t \in \mathbb{R} ; j=1,2, \ldots, k .
$$

We also have the following proposition.

Proposition 4.2. A vector field $X$ on $M$ preserves $\mu$ if and only if (4.8) and (4.9) are satisfied, or, equivalently, each weight $\alpha_{j}$ is a first integral of $X$ and each distribution $\left\{V_{x}{ }^{\alpha_{j}(x)}\right\}$, for $x \in M, j=1,2, \ldots, k$, is preserved by $X$. 
According to foliation theory [10], a field $X$ preserving a foliation is a foliated field.

Suppose a fuzzy subbundle $\mu: T M \rightarrow I$ is $C^{\infty}$ (or smooth). We propose the following definition.

Definition 4.3. The fuzzy subbundle $\mu$ is integrable if

(i) $\left\{V_{x}{ }^{\alpha_{j}(x)}\right\}$ is involutive (or a foliation) $x \in M, j=1,2, \ldots, k$,

(ii) the weight $\alpha_{j}$ is a basic function for the foliation $\left\{V_{x}{ }^{\alpha_{j}(x)}\right\} x \in M$.

Recall that $\alpha: M \rightarrow \mathbb{R}$ is a basic function for a foliation $\mathscr{F}_{F}=\left\{F_{x}\right\}, x \in M$, if for any vector field $X \in \mathscr{F}$ or $\left(X(x) \in F_{x} x \in M\right), \mathscr{L}_{X} \alpha=0$ or $\alpha$ is a first integral of $X$. It means that $\alpha$ is constant on the leaves of $\mathscr{F}^{[}[10]$.

From this definition, we observe that, through $x \in M$ we have a chain of leaves

$$
L_{1} \subset L_{2} \subset \cdots \subset L_{k} \subset M
$$

where $L_{j}$ is the leaf of the foliation $V_{x}{ }^{\alpha_{j}(x)}$ and $\alpha_{j}$ is constant on $L_{j}$. We can use the $\mu$ function to express the above the fact.

REMARK 4.4. It is well known that there are foliations which do not admit any nonconstant basic function [10]. The definition II of integrability ties up closely the weights and the distribution of a fuzzy subspace. This can be relaxed if there is a need for it.

4.3. An extension to integrability II. Finally, we discuss a more general concept of isomorphism of fuzzy subspaces [9]. A possible third integrability condition (INT III) could be based on such an isomorphism (see Remark 4.7 below), and integrability II will then be a special case of INT III.

Let $(U, \mu)$ and $(V, \nu)$ be two fuzzy subspaces. A pair $(L, \theta)$, where $L: U \rightarrow V$ is an isomorphism and $\theta: I \rightarrow I$ is an increasing and onto function, is called an isomorphism of $\mu$ and $v$ if

$$
v(L(u))=\theta(\mu(u)), \quad u \in U
$$

Let $\left\{U^{\alpha_{i}}\right\}, i=1,2, \ldots, k$, and $\left\{V^{\beta_{j}}\right\}, j=1,2, \ldots, l$, be the two flags with weights corresponding $\mu$ and $v$, respectively. From (4.11), we get

$$
\begin{gathered}
k=l \\
\theta\left(\alpha_{i}\right)=\beta_{i}, \quad i=1,2, \ldots, k, \\
L\left(U^{\alpha_{i}}\right)=V^{\beta_{i}} .
\end{gathered}
$$

We now apply this concept of isomorphism to fuzzy subbundle $\mu$ of $T M$. An isomorphism of $\mu$ is given by the following data: a pair $(f, \theta)$ of mappings, 
such that $f: M \rightarrow M$ is a diffeomorphism and

$$
\theta: M \times M \times I \longrightarrow I
$$

is a function which satisfies

(i) $t \longmapsto \theta(x, y, t)$ is increasing and onto, for each pair $x, y \in M$,

(ii) $\mu(d f(\xi))=\theta(\pi(\xi), f(\pi(\xi)), \mu(\xi)), \xi \in T M$,

where $\pi: T M \rightarrow M$ is the bundle projection.

Equivalently, for any $x \in M$,

$$
\mu_{f(x)}(d f(x)(\xi))=\theta\left(x, f(x), \mu_{x}(\xi)\right), \quad \xi \in T_{x} M .
$$

Since we only work with fuzzy subbundles of a given type, we have from (4.12b) and (4.12c) of (4.12)

$$
\begin{gathered}
d f(x)\left(U^{\alpha_{j}(x)}\right)=U^{\alpha_{j}(f(x))}, \\
\alpha_{j}(f(x))=\theta\left(x, f(x), \alpha_{j}(x)\right), \quad j=1,2, \ldots, k .
\end{gathered}
$$

Now, let $X$ be a vector field on $M$ and let $\left\{\phi_{t}\right\},\left\{\tilde{\phi}_{t}\right\}=\left\{d \phi_{t}\right\}$ be the corresponding flows. Suppose $\theta$ is a function as in (4.13) which satisfies (i) and (ii).

Definition 4.5. A vector field $X$ on $M$ preserves $\mu$ with respect to a given $\theta$ if and only if

$$
\mu\left(\tilde{\phi}_{t}(\xi)\right)=\theta\left(\pi(\xi), \phi_{t}(\pi(\xi)), \mu(\xi)\right),
$$

for all $t \in \mathbb{R}, \xi \in T M$, or

$$
\mu_{\phi_{t}(x)}\left(\tilde{\phi}_{t}(x)(\xi)\right)=\theta\left(x, \phi_{t}(x), \mu_{x}(\xi)\right),
$$

for all $t \in \mathbb{R}, \xi \in T_{x} M$ for each $x \in M$.

For the subspaces and weights, we have the following equations:

$$
\begin{gathered}
\tilde{\phi}_{t}(x)\left(U^{\alpha_{j}(x)}\right)=U^{\alpha_{j}\left(\phi_{t}(x)\right)}, \\
\alpha_{j}\left(\phi_{t}(x)\right)=\theta\left(x, \phi_{t}(x), \alpha_{j}(x)\right), \quad x \in M j=1,2, \ldots, k .
\end{gathered}
$$

We note that the choice of a connecting function $\theta$ can be done for every $\tilde{\phi}_{t}, t \in \mathbb{R}$. In the above, we assumed that $\theta$ is the same for all $t$. The second identity (4.19) implies (after passing to differentials)

$$
\mathscr{L}_{X} \alpha_{j}(x)=\mathscr{L}_{X}^{y} \theta\left(x, x, \alpha_{j}(x)\right), \quad j=1,2, \ldots, k,
$$

where $\mathscr{L}_{X}^{y} \theta$ is the Lie derivative of $\theta$ on the second variable, $\theta(x, y, t)$.

Collecting the above equations, we have the following proposition in terms of the flow equations. 
Proposition 4.6. A vector field $X$ on $M$ preserves $\mu$ with respect to $\theta$ if and only if the above equations (4.18) and (4.20) are satisfied.

REMARK 4.7. We get the second integrability condition if $\theta(x, y, t)=t$ for all $x, y \in M, t \in I$. The problem of integrability involving nontrivial $\theta$ function where $\theta(x, y, t)$ is not necessarily equal to $t$ for all $x, y \in M, t \in I$ will be fully discussed in an another paper. Further geometric integration theory and discussions on foliations will follow.

ACKNOWLEDGMENTS. Both authors were supported by the JRC of Rhodes University and the first author was supported by the FRD of South Africa. We gratefully acknowledge the support. We are very thankful and grateful to the anonymous referee for a number of valuable suggestions which improved the paper considerably.

\section{REFERENCES}

[1] K. S. Abdukhalikov, The dual of a fuzzy subspace, Fuzzy Sets and Systems 82 (1996), no. 3, 375-381.

[2] K. S. Abdukhalikov, M. S. Tulenbaev, and U. U. Umirbaev, On fuzzy bases of vector spaces, Fuzzy Sets and Systems 63 (1994), no. 2, 201-206.

[3] R. Forman, Hodge theory and spectral sequences, Topology 33 (1994), no. 3, 591611.

[4] D. Husemoller, Fibre Bundles, McGraw-Hill, New York, 1966.

[5] A. K. Katsaras and D. B. Liu, Fuzzy vector spaces and fuzzy topological vector spaces, J. Math. Anal. Appl. 58 (1977), no. 1, 135-146.

[6] R. Lowen, Convex fuzzy sets, Fuzzy Sets and Systems 3 (1980), no. 3, 291-310.

[7] G. Lubczonok and V. Murali, On flags and fuzzy subspaces of vector spaces, Fuzzy Sets and Systems 125 (2002), no. 2, 201-207.

[8] P. Lubczonok, Fuzzy vector spaces, Fuzzy Sets and Systems 38 (1990), no. 3, 329-343.

[9] B. B. Makamba, Studies in fuzzy groups, Ph.D. thesis, Rhodes University, South Africa, 1992.

[10] P. Molino, Riemannian Foliations, Progress in Mathematics, vol. 73, Birkhäuser Boston, Massachusetts, 1988.

[11] D. Monk, The geometry of flag manifolds, Proc. London Math. Soc. (3) 9 (1959), 253-286.

[12] J. N. Mordeson, Bases of fuzzy vector spaces, Inform. Sci. 67 (1993), no. 1-2, 8792.

[13] T. Morimoto, Geometric structures on filtered manifolds, Hokkaido Math. J. 22 (1993), no. 3, 263-347.

[14] V. Murali and B. B. Makamba, On an equivalence of fuzzy subgroups. I, Fuzzy Sets and Systems 123 (2001), no. 2, 259-264.

V. Murali: Department of Mathematics (Pure and Applied), Rhodes University, Grahamstown 6140, South Africa

E-mail address: v.murali@ru.ac.za

G. Lubczonok: Department of Mathematics (Pure and Applied), Rhodes University, Grahamstown 6140, South Africa

E-mail address: g.7ubczonok@ru.ac.za 


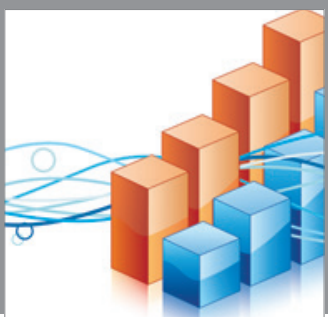

Advances in

Operations Research

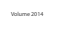

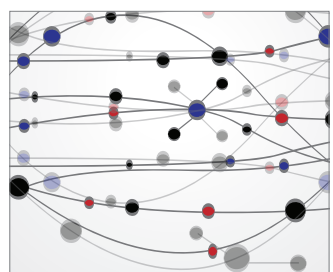

\section{The Scientific} World Journal
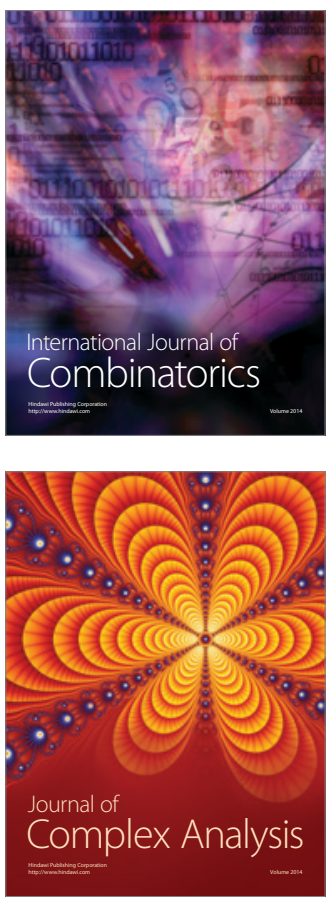

International Journal of

Mathematics and

Mathematical

Sciences
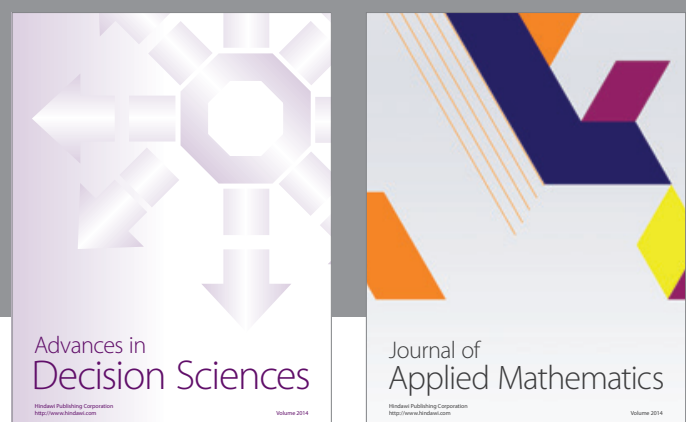

Journal of

Applied Mathematics
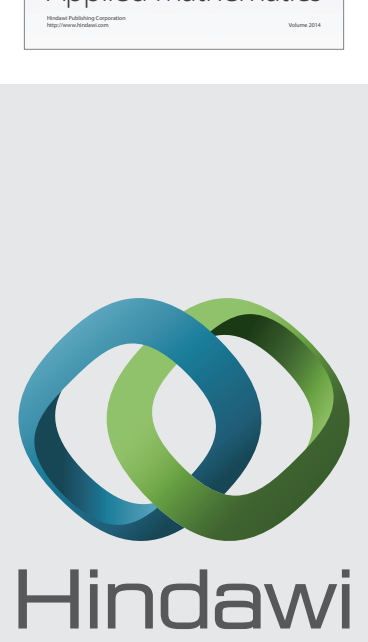

Submit your manuscripts at http://www.hindawi.com
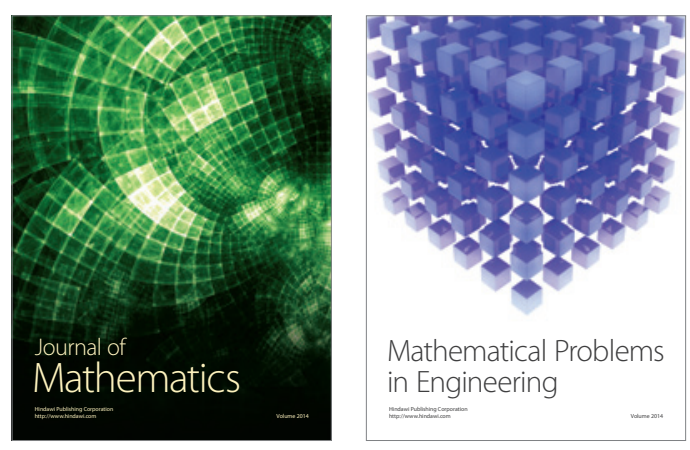

Mathematical Problems in Engineering
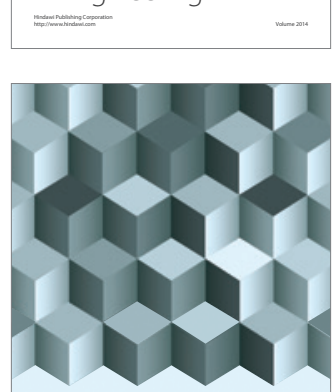

Journal of

Function Spaces
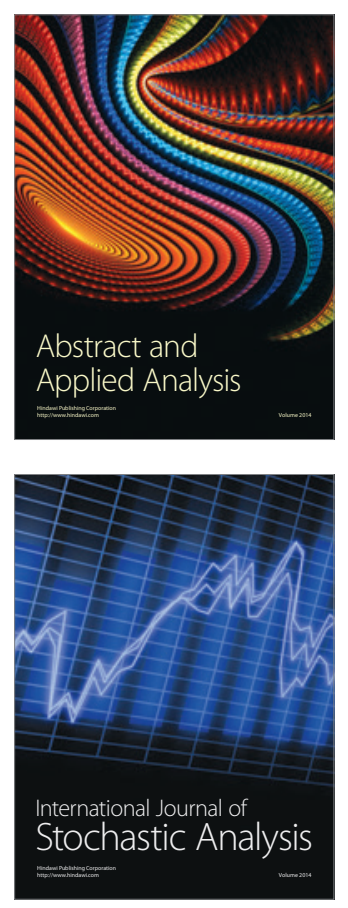

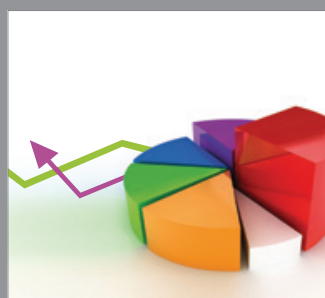

ournal of

Probability and Statistics

Promensencen
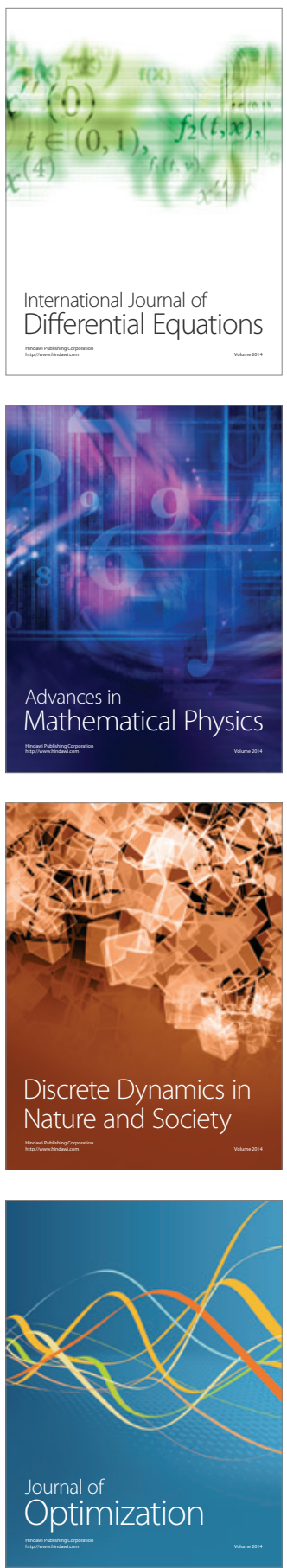